\title{
A High-speed Digital Laser Grating Projection System for the Measurement of 3-dimensional Shapes
}

\author{
Yoon-Chang Park*, Chul Geun Park, Seong Joon Ahn, and Moon-Ho Kang \\ Department of IEC Engineering, Sun Moon University, Tangjeong-myeon, Asan 336-708, Korea \\ Seungjoon Ahn \\ Department of Information Display, Sun Moon University, Tangjeong-myeon, Asan 336-708, Korea
}

(Received May 11, 2009 : revised June 11, 2009 : accepted June 12, 2009)

\begin{abstract}
In the non-contact 3-dimensional (3D) shape measurements, the fringe pattern projection method based on the phase-shifting technique has been considered very effective for its high speed and accuracy. The digital fringe projector in particular has great flexibility in generating fringe patterns since the patterns can be controlled easily by the computer program. In this work, we have developed a high-speed digital laser grating projection system using a laser diode and a polygon mirror, and evaluated its performance. It has been demonstrated that all the optical measurements required to find out the profile of a $3 \mathrm{D}$ object could be carried out within $31 \mathrm{~ms}$, which confirmed the validity of our 3D measurement system. The result implies the more important fact that the speed in 3D measurement can be enhanced remarkably since, in our novel system, there is no device like a LCD or DMD whose response time limits the measurement speed.
\end{abstract}

Keywords: 3D shape measurement, High-speed fringe projector, Laser grating, Phase shifting, Gray code

OCIS codes : (110.0110) Imaging systems; (110.6880) Three-dimensional image acquisition; (120.0120) Instrumentation, measurement, and metrology; (120.6650) Surface measurements; (100.0100) Image processing

\section{INTRODUCTION}

The optical 3-dimensional (3D) measurement technology [1] has expanded its application area rapidly these days. It is used in shape inspection of the product, reverse engineering, computer graphics, electronic industry, and even in the fashion industry $[1,2]$. The technology is so useful and convenient that there have been many efforts made to develop its variations like shadow-moire [3], projection-moire [4], and gray code [5]. These efforts have improved the accuracy and speed of the optical 3D measurement.

To achieve better accuracy, there have been studies to compensate the distortion of the optical system [6-7] and to improve the detector's sensitivity [8] as well as to use better optical sources [8-9]. To reduce the measurement time, researchers have developed key technologies

*Corresponding author: ycpark@sunmoon.ac.kr like rapid processing of image data [11] and combination of optical patterns [12]. Today it is generally accepted that the fringe pattern projection method with the phaseshifting technique is the most effective way to obtain 3D shape with high accuracy and speed [10, 13-15].

The fringe pattern projection system is composed of a fringe pattern projector, a CCD camera, and a control unit. For the fringe projector, there are conventionally two types present. One type adopts a grating element to produce the fringe patterns and the other uses a liquid crystal display (LCD) or digital micromirror device (DMD). When we use the grating element in the fringe pattern projector, the system becomes simple and we can obtain rapid phase shift. But this method is not appropriate for the two-frequency Moire or gray-code system because the alignment of the two grating elements is difficult and the long path of motion of the gratings makes it hard to accomplish real-time measurement.

When the digital projector such as LCD or DMD is 
used to generate patterns $[8,12,15,16]$, there is practically no phase error and it is convenient to change the patterns since the patterns are provided by computer program. However, there is rather long time required to transmit the 2-dimensional image data from the computer to the digital projector, because of the response time of the LCD (or DMD) and the large amount of image data. For many applications, this transmission time is short enough and does not create any problem. But, for a lot of today's delicate applications, the speed of pattern change is not satisfactory for realization of high-speed accurate 3D measurements.

To overcome these shortcomings of the conventional fringe projectors, we propose the novel high-speed digital fringe projection system where a laser diode (LD) and a rotating polygon mirror $(\mathrm{PM})$ produce the fringe grating patterns that can be varied much more rapidly compared with the previous fringe projectors, while keeping all the merits of the conventional digital projectors such as programmable pattern generation and accurate phase shift.

\section{Schematic of Shape Measurement with the LGP System}

Fig. 1 shows the schematic of the Laser Gratings Projector (LGP) developed in this work. The polygon mirror (PM) is composed of 14 evenly-divided reflective facets and makes 10,000 revolutions per minute powered by the high-frequency motor. The angular velocity of the PM can be controlled by the frequency of the external clock that is provided into the motor. The light source is a laser diode (LD) having wavelength of 655 $\mathrm{nm}$ and output power of $30 \mathrm{~mW}$. The laser output is converted to the slit beam by a condenser lens and a rod lens. The focal length of the condenser lens is 4.6 $\mathrm{mm}$ and the diameter of the rod lens is $7 \mathrm{~mm}$. The LD is alternately turned on and off very rapidly by the control signal. When these alternating slit beams are reflected by the revolving PM, they make the fringe grating on the object to be measured.

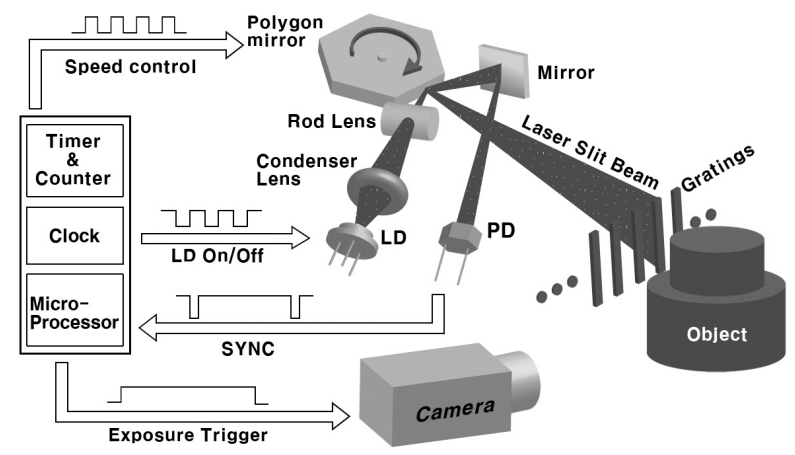

FIG. 1. Schematic diagram of Laser Gratings Projector.
There is a fixed mirror present in the system which reflects the slit beam into the photodiode (PD) when a facet and the laser beam make a certain angle to form the optical path from the LD and the PD through the fixed mirror. Since every facet sends the slit beam to the PD once during a revolution and at the exactly same position, the output current from the PD can be used as the synchronization signal for generation and phase shift of the laser grating.

The pitch of the laser grating, that is, the distance between consecutive fringes can be controlled by adjusting the on-off period of the LD. We can also shift the phase of the grating, which means that the whole grating pattern is shifted horizontally without any other change of its shape. The phase shift can be made by altering the phase of the $\mathrm{LD}$ control signal. That is carried out by the microprocessor (ATMEL, ATMEGA 128). According to the program we already made, the microprocessor delays by a certain amount of time before activating the $\mathrm{LD}$ after recognizing the synchronization signal from the PD, which determines the amount of phase shift.

In fact, the microprocessor brings all information required for generation of grating patterns like on-off period of the LD and the delay time from the PC. During the measurement procedure, the microprocessor sequentially writes down the control parameters on the register of the programmable logic device (PLD) that activates the LD control signal. In a similar way, the microprocessor generates the trigger signal for the CCD camera(Viewworks Co., VM-VGA264C-MC 10) to capture the image.

The CCD camera monitors and captures the image of grating fringes imposed on the object. The CCD camera has a half-inch CCD image sensor with resolution of $640 \times 480$ and can transmit as many as 264 frames/s.

Fig. 2 is the optical geometry of the system for obtaining the shape coordinates. Obtaining these coordinates $\mathrm{s}$ a basic process to measure the $3 \mathrm{D}$ shape of the object. As shown in the figure, the distance from the CCD camera to the reference plane $(Z=0)$ is $\mathrm{L}$

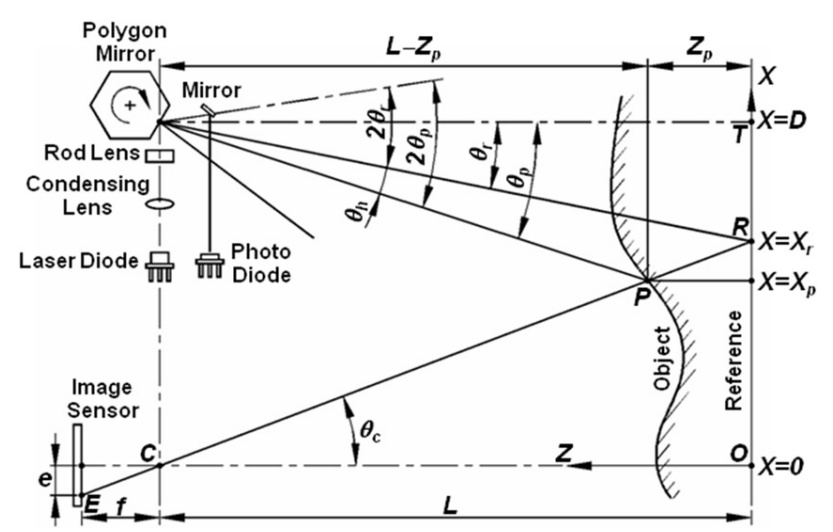

FIG. 2. Optical Geometry of 3D Shape Measurement System. 
and the laser grating projector is as far as $\mathrm{D}$ from the CCD camera. The coordinate $\left(X_{p}, Z_{p}\right)$ of a point $P$ on the object is represented as follows:

$$
X_{P}=\left(L-Z_{P}\right) \frac{e}{f}, \quad Z_{P}=L-\frac{D f}{e+f \tan \theta_{P}}
$$

In equation (1), $f$ is the focal length of the C-mount lens that is installed in front of the CCD image sensor and $e$ is the distance between the center pixel of the image sensor to the image pixel. $\theta_{p}$ is the sum of $\theta_{h}$ and $\theta_{r}$ in the figure and given by equation (2),

$$
\theta_{P}=\theta_{h}+\theta_{r}=\theta_{h}+\tan ^{-1}\left(\frac{D-X_{r}}{L}\right)
$$

Since all the quantities in equation (1) such as $e, f$, $L$, etc. are known except for $\theta_{p}$, we can obtain the shape coordinate $\left(X_{p}, Z_{p}\right)$ of the point $\mathrm{P}$ only if $\theta_{p}$ is found. $\theta_{h}$ can be measured by the phase measuring profilometry (PMP) method that is carried out by our LGP system. Let us denote the angular velocity of the PM as $\omega_{P M}$ and the frequency of the LD control signal as $f_{G}$.

Let $P_{r}$ be the reference phase of the grating at a point $R$ that lies in the reference plane, and $P_{p}$ be the object phase of the grating at a point $P$ that lies in the surface of the object. Then the height of the point $P$ from the reference plane can be obtained from the height phase $P_{h}$ that is defined by $P_{p}-P_{r}$.

Due to the reflection law, the laser beam reflected from the PM scans the object with angular velocity of $2 \omega_{P M}$, that is two times larger than that of the revolving $\mathrm{PM}$. If we represent the angle formed by two adjacent grating fringes as $\theta_{G}, \theta_{G}$ is given as $\theta_{G}=2 \omega_{P M} / f_{G}$. Since $\theta_{h}=\frac{P_{h}}{2 \pi} \theta_{G}$ and $X_{r}=L \frac{e}{f}, \theta_{P}$ can be written as

$$
\theta_{P}=\frac{P_{h}}{2 \pi} \theta_{G}+\tan ^{-1}\left(\frac{f D-e L}{f L}\right) .
$$

Using equations (1) and (3), we can obtain shape coordinate $\left(X_{P}, Z_{P}\right)$ of an arbitrary point $P$ in the surface of the object. We can measure the 3D shape of the object by repeating the process mentioned so far with respect to the sufficiently many surface points of the object. Although the principle of $3 \mathrm{D}$ profilometry in our works can be understood by equations (1) to (3), it is necessary in real measurement to compensate several errors caused by lens distortions, inaccurate values of system parameters, etc. in order to obtain the true shape of the object. Hence we characterize the system ahead of the shape measurement, which will be used to remove the systematic errors through computer-based calibration process [7].

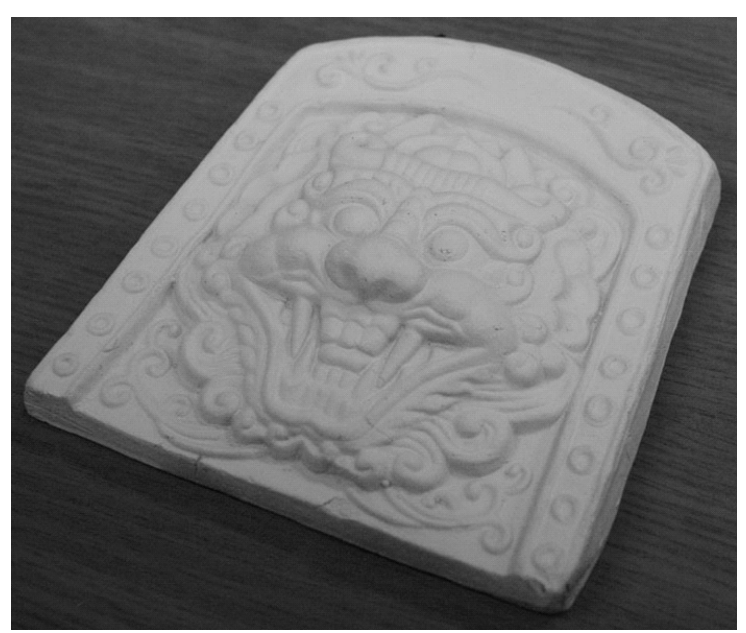

FIG. 3. Photograph of the sample object (W:150 $\times$ $\mathrm{H}: 150 \times \mathrm{D}: 35 \mathrm{~mm})$.

\section{EXPERIMENTAL RESULTS}

We have measured the 3D shape of the object shown in Fig. 3 to evaluate the performance of our LGP system. The object was covered with non-glossy white paint and its dimension was 150 (wide) $\times 150$ (height) $\times 35$ (depth) $\mathrm{mm}^{3}$. The distance $\mathrm{L}$ between the object and the LGP system was $800 \mathrm{~mm}$. The polygon mirror used in the experiment was supported by the air bearings, which kept the revolution very stable.

To measure the 3D shape of the object, six different images needed to be captured, four of which were ordinary fringe grating images laid on the object. The phase difference between successive laser grating patterns was $90^{\circ}$. The frequency of the main clock in Fig. 2 was $40 \mathrm{MHz}$. In the PMP method, the LD was alternately turned on and off every other 56 clocks. The images with respect to each of the illuminated laser grating are shown in Fig. 4(a) $\sim 4(\mathrm{~d})$. From these four images $\left(\mathrm{IO}_{0} \sim \mathrm{IO}_{270}\right)$, we could obtain the Moire phase map [17] of the object that is shown in Fig. 5(a) where the Moire phase is a wrapped phase that ranges between $-\pi$ and $+\pi$. Therefore the Moire phase has a problem of $2 \pi$-ambiguity that makes it impossible to determine the value of the height phase $P_{h}$ in equation (3).

To eliminate the $2 \pi$-ambiguity in the Moire phase map, we captured two additional laser grating images laid on the object where the illuminated patterns were 2-bit gray code patterns. In gray code patterns, the pitch of the gray-coded laser grating was four times larger than that of ordinary laser gratings. Consequently the on-off period of the $\mathrm{LD}$ was also four times larger, that is, the of-off period of the LD was 224 clocks. Using the images obtained by the gray code that are shown in Fig. $4(\mathrm{e})$ and $4(\mathrm{f})$, we could eliminate the $2 \pi$-ambiguity and get a correct map of the unwrapped height phase $P_{h}[18]$ that is represented by Fig. 5(b). 


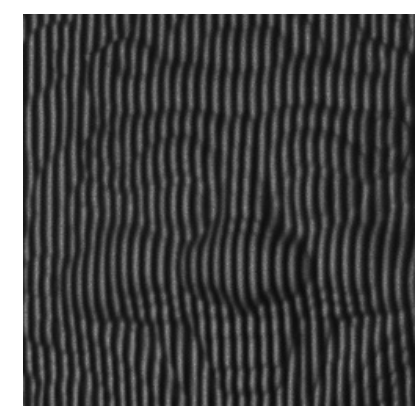

(a) phase shift $0^{\circ}, \mathrm{IO}_{0}$

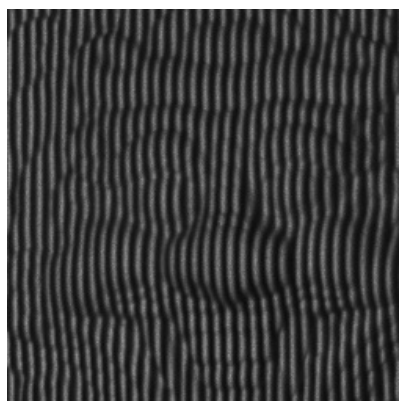

(c) phase shift $180^{\circ}, \mathrm{IO}_{180}$

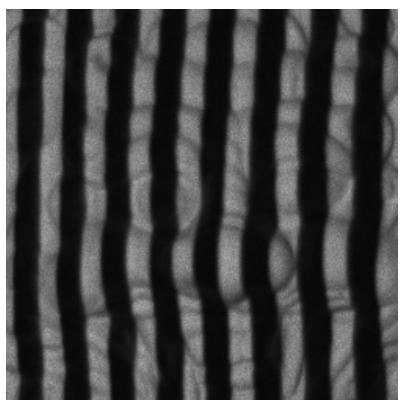

(e) first gray code, $\mathrm{IG}_{1}$

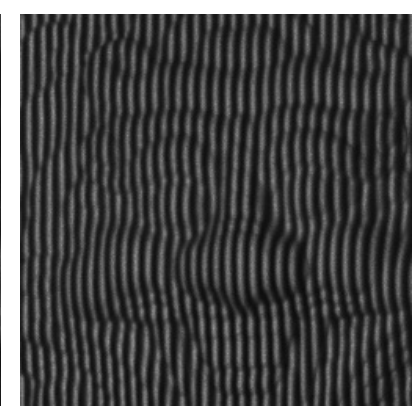

(b) phase shift $90^{\circ}, \mathrm{IO}_{90}$

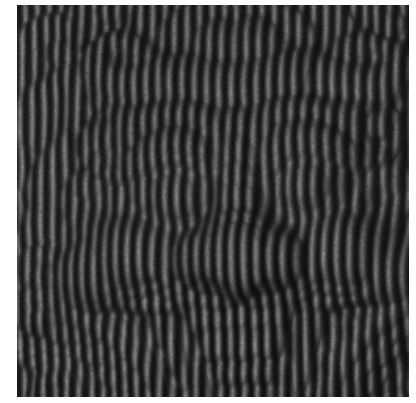

(d) phase shift $270^{\circ}, \mathrm{IO}_{270}$

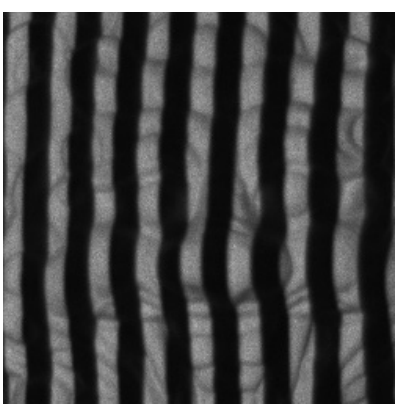

(f) second gray code, $\mathrm{IG}_{2}$
FIG. 4. The images of the laser gratings illuminated on the object.

Since we got the map of $P_{h}$, we could obtain the 3D shape (Fig. $5(\mathrm{c})$ ) of the object by calculating the shape coordinates $\left(X_{P}, X_{P}\right)$ all over the object using equations (1) and (3). As shown in the figure, the 3D shape measured by our LGP system is in good agreement with the real object, which proves the validity of our 3D measurement system. The periodic fine stripes in Fig. 5(c) are noise caused by non-sinusoidal intensity profiles of the laser fringe gratings [15].

As previously mentioned, the alteration of fringe patterns takes place by writing down the control parameters that determine the pitch and phase into the PLD's register. Because this process needs just a few microseconds and is carried out during the LD is turned on to generate the synchronization signal, we do not require any extra time to change the patterns. That means it is possible to illuminate as many as $\sim 2300$ grating patterns in one second, each of which has different pitch and

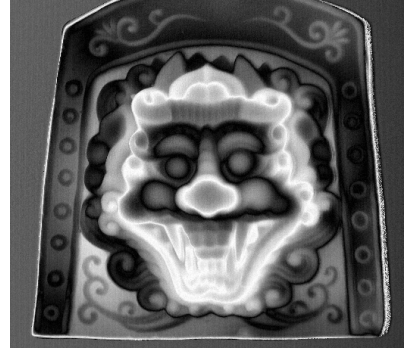

(a) Moire phase map

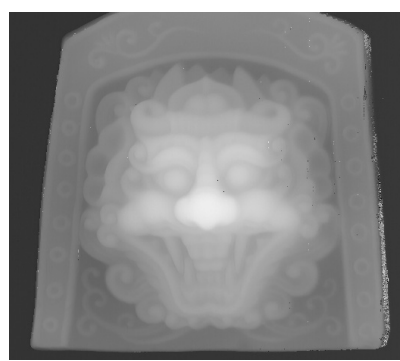

(b) Height phase map

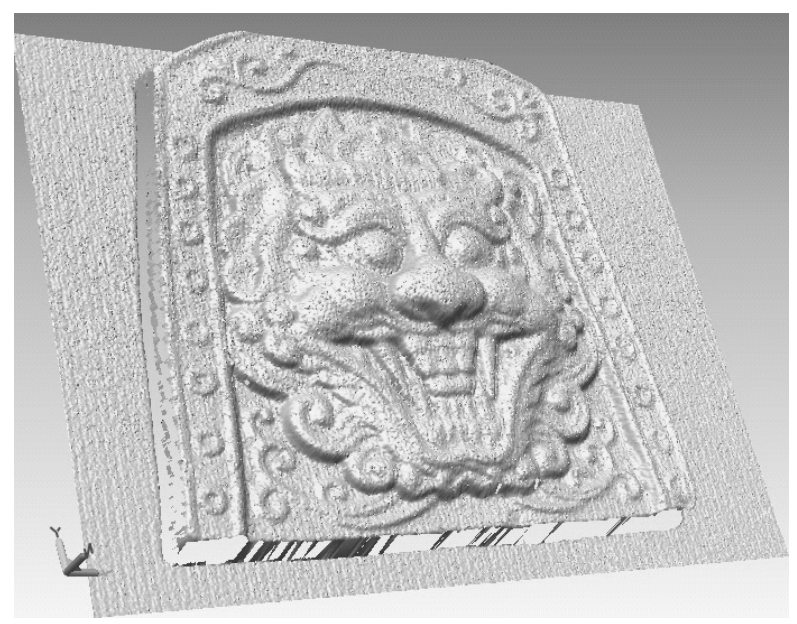

(c) Reconstructed 3D geometry

FIG. 5. Phase Map \& Reconstructed 3D geometry.

phase from each other, which is a remarkable improvement compared with performance of the conventional digital projector system using LCD or DMD where the maximum pattern change in a second is limited to $\sim 300$.

In this work, we kept the same laser grating patterns while 12 facets encountered the laser beam because of the low output power of the laser diode and low sensitivity of the CCD image sensor. Hence it took $\sim 31 \mathrm{~ms}$ to get the six CCD images of Fig. 4. With the LD and CCD camera having sufficiently high output power and sensitivity, respectively, we can capture one image for one facet, which means that all the six images required to reconstruct the $3 \mathrm{D}$ shape of the object can be acquired within $2.6 \mathrm{~ms}$.

\section{CONCLUSIONS}

In this work, we have developed the high-speed digital laser grating projection system for measurement of the 3D shape of the object. In our LGP system, the laser diode and the polygon mirror were used to generate the various fringe grating patterns. Since the pitch and phase of the laser grating can be changed by simply adjusting the LD on/off period and the delay time in the microprocessor, it takes just a few microseconds to change 
the patterns. In contrast, the LCD or DMD projector system needs several milliseconds to transmit other grating patterns. Our LGP system, in principle, can measure the $3 \mathrm{D}$ shape of an object within $2.6 \mathrm{~ms}$, which is great improvement compared with the conventional digital projection systems.

We have proved the validity of our LGP system through the experiment where the six different laser grating patterns were used to measure the accurate $3 \mathrm{D}$ shape of the object. We expect that our LGP system and measurement method can be a very convenient tool for the 3D shape measurement, especially for the applications that need rapid measurement.

\section{ACKNOWLEDGMENT}

This research was supported by the MKE, Korea, under the ITRC support program supervised by the IITA (IITA-2008-C1090-0801-0013).

\section{REFERENCES}

1. F. Chen, G. M. Brown, and M. Song, "Overview of threedimensional shape measurement using optical methods," Opt. Eng. 39, 10-22 (2000).

2. J. W. Kang and C. K. Hong, "Three dimensional shape measurement of a micro Fresnel lens with in-line phaseshifting digital holographic microscopy," J. Opt. Soc. Korea 10, 178-183 (2006).

3. T. Yoshizawa and T. Tomosawa, "Shadow moire topography by means of phase-shift method,” Opt. Eng. 32, 1668-1674 (1993).

4. W. J. Ryu, Y. J. Kang, S. H. Baik, and S. J. Kang, “A study on the 3-D measurement by using digital projection moire method," Optik 119, 453-458 (2008).

5. R. C. Daley and L. G. Hassebrook, "Channel capacity model of binary encoded structured light-stripe illumination,” Appl. Opt. 37, 3689-3696 (1998).
6. A. G. Wiley and K. W. Wong, "Geometric calibration of zoom lenses for computer vision metrology," Photogramm. Eng. Remote Sens. 61, 69-74 (1995).

7. L. C. Chen and C. C. Liao, "Calibration of 3D surface profilometry using digital fringe projection," Meas. Sci. Technol. 16, 1554-1566 (2005).

8. J. Pan, P. S. Huang, and F. P. Chiang, "Color phaseshifting technique for three-dimensional shape measurement," Opt. Eng. 45, 013602-1-9 (2006).

9. H. Liu, G. Lu, S. Wu, S. Yin, and F. T. S. Yu, "Speckleinduced phase error in laser-based phase-shifting projected fringe profilometry,” J. Opt. Soc. Am. 16, 1484-1495(1999).

10. C. Xiaobo, X. J. Tong, J. Tao, and J. Ye, "Research and development of an accurate $3 \mathrm{D}$ shape measurement system based on fringe projection: model analysis and performance evaluation," Precision Eng. 32, 215-221 (2008).

11. S. Zhang, D. Royer, and S. T. Yau, "GPU-assisted highresolution, real-time 3-D shape measurement," Opt. Exp. 14, 9120-9129 (2006).

12. F. Tsalakanidou, F. Forster, S. Malassiotis, and M. G. Strintzis, "Real-time acquisition of depth and color images using structured light and its application to 3D face recognition,” Real-Time Imaging 11, 358-369 (2005).

13. D. Kim, B. J. Baek, Y. D. Kim, and B. Javidi, "3D nano object recognition based on phase measurement technique," J. Opt. Soc. Korea 11, 108-112 (2007).

14. D. Kim and Y. J. Cho, "3-D surface profile measurement using an acousto-optic tunable filter based spectral phase shifting technique,” J. Opt. Soc. Korea 12, 281-287 (2008).

15. S. Zhang and P. S. Huang, "Phase error compensation for a 3-D shape measurement system based on the phaseshifting method," Opt. Eng. 46, 063601-1-9 (2007).

16. S. Kakunai, T. Sakamoto, and K. Iwata, "Profile measurement taken with liquid-crystal gratings," Appl. Opt. 38, 2824-2828 (1999).

17. K. Jeong, Y. Park, and K. Park, "A study on the phase measuring profilometry with parallel-optical axes," KSPE 17, 210-217 (2000).

18. G. Sansoni, M. Carocci, and R. Rodella, "Three-dimensional vision based on a combination of gray-code and phaseshift light projection: analysis and compensation of the systematic errors," Appl. Opt. 38, 6565-6573 (1999). 